\title{
Macitentan in pulmonary hypertension due to left ventricular dysfunction
}

\author{
Jean-Luc Vachiéry ${ }^{1}$, Marion Delcroix (1) ${ }^{2}$, Hikmet Al-Hiti ${ }^{3}$, Michela Efficace ${ }^{4}$, \\ Martin Hutyra ${ }^{5}$, Gabriela Lack ${ }^{6}$, Kelly Papadakis ${ }^{7}$ and Lewis J. Rubin ${ }^{8}$
}

Affiliations: ${ }^{1}$ Dept of Cardiology, Hôpital Erasme, Université Libre de Bruxelles, Brussels, Belgium. ${ }^{2}$ Dept of Respiratory Diseases, KU Leuven - University Hospitals of Leuven, Leuven, Belgium. ${ }^{3}$ Dept of Cardiology, Institute of Clinical and Experimental Medicine-IKEM, Prague, Czech Republic. ${ }^{4}$ Dept of Clinical Development - Biostatistics, Actelion Pharmaceuticals Italia, Imperia, Italy. ${ }^{5}$ Dept of Internal Medicine I - Cardiology, University Hospital Olomouc, Olomouc, Czech Republic. ${ }^{6}$ Dept of Global Clinical Science \& Epidemiology, Actelion Pharmaceuticals Ltd, Allschwil, Switzerland. ${ }^{7}$ Dept of Clinical Research, Actelion Clinical Research Inc., Cherry Hill, NJ, USA. ${ }^{8}$ Division of Pulmonary and Critical Care Medicine, University of California San Diego, La Jolla, CA, USA.

Correspondence: Jean-Luc Vachiéry, Dept of Cardiology, Hôpital Erasme, Université Libre de Bruxelles, 808 Route de Lennik, 1070 Brussels, Belgium. E-mail: jeanluc.vachiery@erasme.ulb.ac.be

@ERSpublications

In combined post- and pre-capillary $\mathrm{PH}$ patients, macitentan was associated with increased incidence of significant fluid retention versus placebo http://ow.ly/leop30hBkQm

Cite this article as: Vachiéry J-L, Delcroix M, Al-Hiti H, et al. Macitentan in pulmonary hypertension due to left ventricular dysfunction. Eur Respir J 2018; 51: 1701886 [https://doi.org/10.1183/13993003.018862017].

ABSTRACT The MELODY-1 study evaluated macitentan for pulmonary hypertension because of left heart disease (PH-LHD) in patients with combined post- and pre-capillary $\mathrm{PH}$.

63 patients with $\mathrm{PH}-\mathrm{LHD}$ and diastolic pressure gradient $\geqslant 7 \mathrm{mmHg}$ and pulmonary vascular resistance $($ PVR $)>3 W U$ were randomised to macitentan $10 \mathrm{mg}(\mathrm{n}=31)$ or placebo $(\mathrm{n}=32)$ for 12 weeks. The main end-point assessed a composite of significant fluid retention (weight gain $\geqslant 5 \%$ or $\geqslant 5 \mathrm{~kg}$ because of fluid overload or parenteral diuretic administration) or worsening in New York Heart Association functional class from baseline to end of treatment. Exploratory end-points included changes in N-terminal pro-brain natriuretic peptide (NT-proBNP) and haemodynamics at week 12.

Seven macitentan-treated and four placebo-treated patients experienced significant fluid retention/ worsening functional class; treatment difference, $10.08 \%$ (95\% CI $-15.07-33.26 ; \mathrm{p}=0.34$ ). The difference, driven by the fluid retention component, was apparent within the first month. At week 12, versus placebo, the macitentan group showed no change in PVR, mean right atrial pressure or pulmonary arterial wedge pressure; a non-significant increase in cardiac index (treatment effect $0.4(95 \% \mathrm{CI} 0.1-0.7) \mathrm{L} \cdot \mathrm{min}^{-1} \cdot \mathrm{m}^{-2}$ ) and decrease in NT-proBNP (0.77 (0.55-1.08)) was observed. Adverse events and serious adverse events were numerically more frequent with macitentan versus placebo.

Macitentan-treated patients were quantitatively more likely to experience significant fluid retention versus placebo. Macitentan resulted in no significant changes in any exploratory end-points.

Editorial comment in Eur Respir J 2018; 51: 1702589

This article has supplementary material available from erj.ersjournals.com

Received: Sept 152017 | Accepted after revision: Nov 202017

Support statement: This study was supported by Actelion Pharmaceuticals Ltd. Funding information for this article has been deposited with the Crossref Funder Registry.

Conflict of interest: Disclosures can be found alongside this article at erj.ersjournals.com

Copyright OERS 2018 


\section{Introduction}

Pulmonary hypertension $(\mathrm{PH})$ is a common complication of heart failure (HF) [1]. Classified as group 2 $\mathrm{PH}$ (post-capillary $\mathrm{PH}$ ), $\mathrm{PH}$ due to left heart disease (PH-LHD) is associated with significant morbidity and poor prognosis [2]. Initially triggered by a passive increase in left atrial pressure, $\mathrm{PH}$ may further progress in response to a series of pathobiological events that may contribute to vascular remodelling and right $\mathrm{HF}$, including endothelial dysfunction, vasoconstriction and deregulation of several mechanisms $[1,3]$.

The haemodynamic classification of post-capillary $\mathrm{PH}$ has been extensively discussed and debated. Although recently challenged, the current European Society of Cardiology (ESC) and the European Respiratory Society (ERS) guidelines define two types of post-capillary $\mathrm{PH}$ : 1) isolated post-capillary $\mathrm{PH}$ (IpcPH; diastolic pressure gradient (DPG) $<7 \mathrm{mmHg}$ and/or pulmonary vascular resistance (PVR) $<3$ Wood units (WU)) and 2) post-capillary $\mathrm{PH}$ with a pre-capillary component (termed combined post- and pre-capillary $\mathrm{PH}(\mathrm{CpcPH})$; elevated $\mathrm{DPG} \geqslant 7 \mathrm{mmHg}$ and/or elevated $\mathrm{PVR}>3 \mathrm{WU})$ [2]. Compared with $\mathrm{IpcPH}$, it has been suggested that $\mathrm{CpcPH}$ may be associated with a worse prognosis in patients with $\mathrm{PH}$ induced by $\mathrm{HF}[2,4]$.

In patients with HF, plasma endothelin-1 levels are elevated and are associated with increased pulmonary pressure [5] and a greater risk of mortality [6]. Based on this observation, endothelin receptor antagonists (ERAs) have been evaluated as a potential treatment option for HF; a potential benefit has not been demonstrated, possibly because of a high incidence of premature study withdrawal caused by early excess fluid retention and worsening HF [7-9]. However, to date, no study has stratified patients according to the presence and severity of PH-LHD. Given their mode of action, ERAs may be beneficial in the subset of patients with PH-LHD with a pre-capillary component.

Macitentan is an ERA approved for the long-term treatment of pulmonary arterial hypertension (PAH) [10]. Overall, oedema is not a significant safety concern with macitentan in patients with PAH [11], thereby making it a suitable candidate for evaluation in PH-LHD. Owing to the potential risk of fluid retention with ERAs in the setting of LHD, the primary objective of MELODY-1 (Macitentan in subjects with combined prE- and post-capiLlary pulmOnary hypertension due to left ventricular DYsfunction) was to evaluate the safety and tolerability of macitentan $10 \mathrm{mg}$ in patients with $\mathrm{CpcPH}$. This is the first study to evaluate an ERA specifically in this patient population.

\section{Methods}

\section{Patient population}

Patients aged $\geqslant 18$ years with $\mathrm{CpcPH}$ confirmed by right heart catheterisation (RHC) (mean pulmonary arterial pressure $\geqslant 25 \mathrm{mmHg}$, pulmonary arterial wedge pressure (PAWP) $>15 \mathrm{mmHg}$ and $<25 \mathrm{mmHg}$, $\mathrm{PVR}$ at rest $\geqslant 3 \mathrm{WU}\left[\geqslant 240 \mathrm{dyn} \cdot \mathrm{s} \cdot \mathrm{cm}^{-5}\right]$ and $\mathrm{DPG} \geqslant 7 \mathrm{mmHg}$ ) within 12 weeks prior to or during screening were eligible. Patients were required to have a left ventricular ejection fraction (LVEF) $\geqslant 30 \%$, as measured by echocardiography during screening and New York Heart Association Functional Class (NYHA FC) II or III, a 6 -min walk distance $(6 \mathrm{MWD}) \geqslant 150 \mathrm{~m}$ and an optimised stable dose of oral diuretic(s) for at least 1 week prior to the RHC and up to randomisation. Patients receiving ERAs, prostacyclin analogues, phosphodiesterase type-5 inhibitors or soluble guanylate cyclase stimulators were excluded.

Full eligibility criteria are provided in table S1. Written informed consent was obtained from all patients. The trial adhered to the Declaration of Helsinki and the research protocol was approved by local institutional review boards or independent ethics committees.

\section{Study design}

MELODY-1 was a multicentre, double-blind, randomised, placebo-controlled, 12-week, phase II study (NCT02070991). Following screening, patients were randomly assigned (1:1) to receive macitentan $10 \mathrm{mg}$ or placebo once daily, stratified by the LVEF at baseline $(<50 \%$ versus $\geqslant 50 \%)$. Patients were assessed at randomisation and at weeks $1,4,8$ and 12 . Patients who prematurely discontinued study treatment were required to have their end of treatment (EOT) visit within 7 days after discontinuation. All patients were to have a safety follow-up visit of at least 30 days after permanent discontinuation of study treatment (end of study visit). Adverse events (AEs) were monitored throughout the study.

\section{Outcome measures}

The main study end-point, related to safety, assessed a composite of significant fluid retention or worsening in NYHA FC from baseline up to the EOT. Significant fluid retention was defined as an increase from baseline in body weight at any time by $\geqslant 5 \%$ or $\geqslant 5 \mathrm{~kg}$ due to fluid overload or parenteral administration of diuretics. Other safety end-points included the evaluation of treatment-emergent AEs and laboratory abnormalities up to 30 days after study treatment discontinuation and the change in 
diuretic therapy (i.e. increase in dose or additional administration) up to the EOT. Exploratory efficacy end-points included PVR and N-terminal pro-brain natriuretic peptide (NT-proBNP) levels, both at week 12 expressed as a percentage of baseline, the change from baseline to week 12 in other haemodynamic parameters, echocardiographic parameters and $6 \mathrm{MWD}$, and the occurrence of hospitalisations for worsening of HF up to the EOT. To ensure quality of all RHCs, training on the study RHC guidelines was provided and RHC tracings from the first patients randomised at each site were reviewed by the steering committee and if necessary, additional tracings were reviewed.

\section{Statistical methods}

Owing to the exploratory nature of the study, there was no formal power calculation. With 30 patients per treatment group, a difference of at least $30 \%$ in proportions between macitentan and placebo for the main safety end-point can be ruled out with a statistical confidence of $95 \%$.

The main safety end-point was analysed on the safety analysis set (all randomised patients who received at least one dose of study treatment). The proportion of patients with significant fluid retention or worsening in FC up to the EOT is presented for each treatment group with the two-sided $95 \%$ confidence intervals (CIs) and the treatment difference reported with the two-sided 95\% CIs based on the exact binomial distribution and a p-value based on the Fisher's exact test. Other safety and tolerability end-points (AEs, serious AEs, discontinuations due to AEs and laboratory measurements) were presented descriptively.

Efficacy analyses were based on the full analysis set (all randomised patients) evaluating observed cases at baseline and week 12 (time window +5 days). Missing values in the week 12 time window are not imputed for the main analysis. Additional analyses for missing data at week 12 for haemodynamic variables were applied based on the last observation carried forward for patients still alive and not hospitalised, whereas the worst value was imputed for patients who died or were hospitalised for HF. The patient's worst value was taken as their baseline value corrected with the highest percentage of worsening from baseline observed in any patient within the same treatment group. PVR and NT-proBNP data at week 12 were expressed as a percentage of baseline, and presented with geometric means and 95\% CIs for each treatment group, and with the ratio of geometric means (95\% CIs) for the treatment effect of macitentan versus placebo. Other haemodynamic and echocardiographic parameters and 6MWD are presented with mean (standard deviation (SD)) for each treatment group and with mean (95\% CIs) absolute change from baseline to week 12 for the treatment difference. Statistical analyses were conducted using Statistical Analysis System version 9.3 (SAS Institute, Cary, NC, USA).

\section{Results}

\section{Patients}

Patients were screened in 28 sites across 11 countries from June 2014 to July 2015. A total of 63 patients were randomised to macitentan $(n=31)$ or placebo $(n=32)$ (figure 1). The median (range) treatment duration was $12.0(0.3-14.9)$ weeks in the macitentan and $12.1(8.0-14.1)$ weeks in the placebo group. $11(17.5 \%)$ patients prematurely discontinued treatment: seven in the macitentan and four in the placebo group. Of the 63 randomised patients, $28(90.3 \%)$ randomised to macitentan and 32 (100\%) randomised to placebo completed the 30-day safety follow-up.

Overall, the median age was 71.0 years, $87.3 \%$ of patients were aged $\geqslant 65$ years, and the median body mass index was $32.4 \mathrm{~kg} \cdot \mathrm{m}^{-2}$ (table 1 ). The majority of patients $(76.2 \%)$ had a preserved LVEF (i.e. $\left.\geqslant 50 \%\right)$ and rate-controlled atrial fibrillation $(73.0 \%)$ at baseline. At baseline, the population had a median PVR of $462.0 \mathrm{dyn} \cdot \mathrm{s} \cdot \mathrm{cm}^{-5}$, a median PAWP of $20.0 \mathrm{mmHg}$, and a median mean right atrial pressure (mRAP) of $13.0 \mathrm{mmHg}$ (table 2). Baseline median NT-proBNP and 6MWD were at $1515 \mathrm{pg} \cdot \mathrm{mL}^{-1}$ and $300 \mathrm{~m}$, respectively. A greater proportion of patients in the macitentan group was female and in FC III than the placebo group. Other baseline parameters were balanced between the groups.

\section{Main study end-point}

Seven $(22.6 \%)$ patients in the macitentan group and 4 (12.5\%) in the placebo group experienced the main composite end-point (table 3). The treatment difference was $10.08 \%$ (95\% CI) -15.07-33.26) and was driven by the fluid retention component (treatment difference $13.21 \%$ (95\% CI -11.96-36.21]). In the macitentan group, all seven patients who met the main safety end-point experienced significant fluid retention, with one patient also reporting worsening in FC. Three out of the four patients in the placebo group met this safety end-point due to significant fluid retention and one patient met the safety end-point because of worsening in FC from II to III.

In the macitentan group, the main end-point was reported in six out of $25(24.0 \%)$ patients with a baseline $\mathrm{LVEF} \geqslant 50 \%$ and in one out of six (16.7\%) patients with a baseline LVEF $<50 \%$. In the placebo 


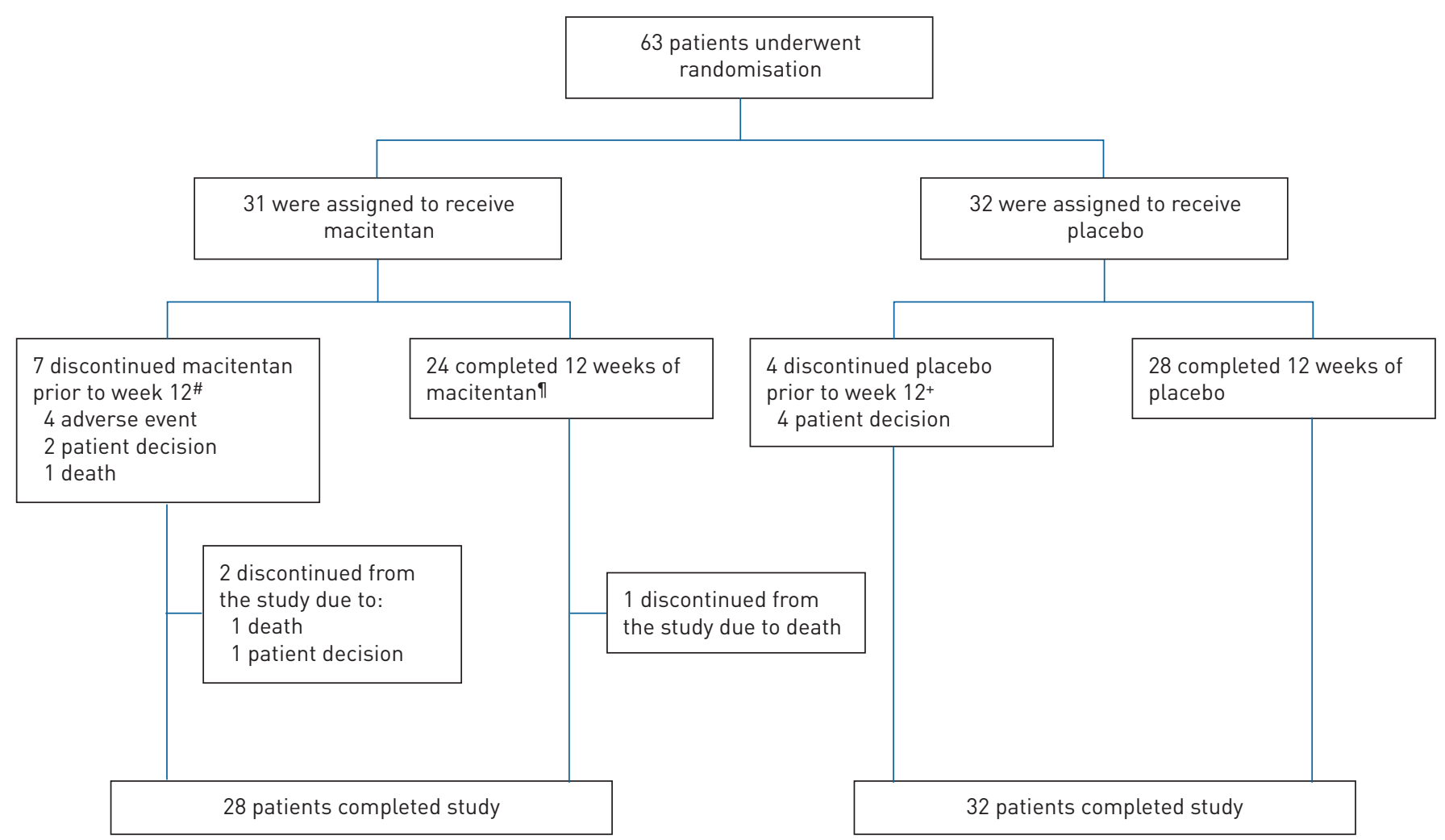

FIGURE 1 Patient disposition. \#: of the seven patients who discontinued treatment with macitentan prior to week 12 , five went on to have their end of study visit and were considered to have completed the study. The remaining two patients did not have an end of study visit (death $n=1$; patient decision $n=1$ ). " : of the 24 patients who completed 12 weeks of macitentan, one did not have an end of study visit (death $n=1$ ); ${ }^{+}$: all four patients who discontinued placebo prior to week 12 had their end of study visit and were considered to have completed the study.

group, the main end-point was reported by three out of $23(13.0 \%)$ and one out of nine (11.1\%) patients with a baseline $\mathrm{LVEF} \geqslant 50 \%$ and $<50 \%$, respectively.

In the macitentan group, four out of seven patients experienced significant fluid retention within the first month of randomisation (days 10, 12, 28, 29); for the three other patients, fluid retention occurred on days 57, 57 and 65. In the placebo group, three patients experienced fluid retention on days 43, 50 and 61.

\section{Other safety/tolerability end-points}

$23(74.2 \%)$ patients in the macitentan group and 19 (59.4\%) in the placebo group experienced at least one AE. A numerically higher number of patients in the macitentan group experienced at least one serious $\mathrm{AE}$ versus the placebo group (11 (35.5\%) versus six $(18.8 \%))$ and discontinued treatment prematurely due to AEs (5 [16.1\%] versus 0 ); these differences were driven by patients who met the main study end-point (tables S2 and S3). Eight (25.8\%) patients in the macitentan group and 6 (18.8\%) in the placebo group had at least one AE related to oedema and fluid overload (table S4).

Overall, two patients died, both in the macitentan group: one patient died 1 day after receiving the first dose of macitentan due to respiratory failure primarily caused by bacterial respiratory tract infection, and one patient died 16 days after having completed the 12-week treatment period, reported as sudden death. Both deaths were considered to be unrelated to study treatment by the treating physician.

Laboratory measurements did not identify any new safety signals (table S5). Haemoglobin values $<100 \mathrm{~g} \cdot \mathrm{L}^{-1}$ were reported in five patients $(17.9 \%)$ in the macitentan group and two $(6.3 \%)$ in the placebo group; among these patients, two in the macitentan and one in the placebo group experienced the main end-point. Nine (29.0\%) patients in the macitentan group and seven $(21.9 \%)$ in the placebo group had a change in diuretic therapy up to EOT. In most cases, the change in diuretic administration was related to an AE (eight out of nine and six out of seven patients in the macitentan and placebo groups, respectively).

\section{Haemodynamic and echocardiographic parameters}

No significant changes in haemodynamic variables were observed with macitentan versus placebo (table 4, table S6). Improvements in PVR, transpulmonary pressure gradient (TPG), DPG and mean pulmonary 


\begin{tabular}{|c|c|c|c|}
\hline & Macitentan & Placebo & Total \\
\hline Subjects $\mathrm{n}$ & 31 & 32 & 63 \\
\hline Median (IQR) age years & $70.0(67.0-73.0)$ & $72.0(68.0-75.5)$ & $71.0(67.0-75.0)$ \\
\hline Age $\geqslant 65$ years $n(\%)$ & $26(83.9)$ & $29(90.6)$ & 55 (87.3) \\
\hline Female sex $n(\%)$ & $25(80.6)$ & $16(50.0)$ & $41(65.1)$ \\
\hline Median (IQR) BMI kg. $\mathrm{m}^{-2}$ & $33.3(28.8-38.3)$ & $31.2(27.6-34.5)$ & $32.4(28.7-36.0)$ \\
\hline \multicolumn{4}{|l|}{ Geographic region $\mathrm{n}(\%)$} \\
\hline Europe and Israel & 28 (90.3) & $24(75.0)$ & 52 (82.5) \\
\hline North America & 3 (9.7) & $8(25.0)$ & $11(17.5)$ \\
\hline \multicolumn{4}{|l|}{ Specific medical conditions $n(\%)^{\Uparrow}$} \\
\hline Atrial fibrillation & 22 (71.0) & $24(75.0)$ & $46(73.0)$ \\
\hline Diabetes mellitus type 2 & $14(45.2)$ & $13(40.6)$ & $27(42.9)$ \\
\hline Dyslipidaemia & $3(9.7)$ & $2(6.3)$ & $5(7.9)$ \\
\hline Hypercholesterolaemia & $8(25.8)$ & $5(15.6)$ & $13(20.6)$ \\
\hline Hyperlipidaemia & $3(9.7)$ & $4(12.5)$ & $7(11.1)$ \\
\hline Obesity (BMI $>30 \mathrm{~kg} \cdot \mathrm{m}^{-2}$ ) & $20(64.5)$ & $20(62.5)$ & $40(63.5)$ \\
\hline \multicolumn{4}{|l|}{ Renal impairment $\mathrm{n}(\%)$} \\
\hline Moderate $^{+}$ & $14(45.2)$ & $11(34.4)$ & 25 (39.7) \\
\hline None/mild ${ }^{\S}$ & $17(54.8)$ & $21(65.6)$ & $38(60.3)$ \\
\hline Right heart failure $\mathbf{n}(\%)^{f}$ & $7(22.6)$ & $11(34.4)$ & $18(28.6)$ \\
\hline Systemic hypertension n (\%) & 30 (96.8) & $27(84.4)$ & 57 (90.5) \\
\hline \multicolumn{4}{|l|}{ LVEF n $(\%)$} \\
\hline$<50 \%$ & $6(19.4)$ & $9(28.1)$ & $15(23.8)$ \\
\hline$\geqslant 50 \%$ & $25(80.6)$ & $23(71.9)$ & $48(76.2)$ \\
\hline \multicolumn{4}{|l|}{ Concomitant medications $\mathrm{n}(\%)$} \\
\hline ACE inhibitors & $7(22.6)$ & $9(28.1)$ & $16(25.4)$ \\
\hline Angiotensin II antagonists & 13 (41.9) & $11(34.4)$ & $24(38.1)$ \\
\hline Beta blockers & $24(77.4)$ & $19(59.4)$ & $43(68.3)$ \\
\hline Thiazide diuretics & $8(25.8)$ & $8(25.0)$ & $16(25.4)$ \\
\hline Aldosterone antagonists & $15(48.4)$ & $11(34.4)$ & $26(41.3)$ \\
\hline Loop diuretics & 30 (96.8) & $29(90.6)$ & 59 (93.7) \\
\hline Vitamin $\mathrm{K}$ antagonists & $15(48.4)$ & 20 (62.5) & $35(55.6)$ \\
\hline HMG CoA reductase inhibitors & $12(38.7)$ & $16(50.0)$ & $28(44.4)$ \\
\hline Calcium-channel blockers & $9(29.0)$ & $9(28.1)$ & $18(28.6)$ \\
\hline Platelet aggregation inhibitors & $8(25.8)$ & $14(43.8)$ & 22 (34.9) \\
\hline Biguanides & $6(19.4)$ & $5(15.6)$ & $11(17.5)$ \\
\hline Inhibitors of uric acid production & $10(32.3)$ & $17(53.1)$ & $27(42.9)$ \\
\hline
\end{tabular}

IQR: interquartile range; BMI: body mass index; LVEF: left ventricular ejection fraction; ACE: angiotensin-converting enzyme; HMG CoA reductase inhibitors: 3-hydroxy-3-methyl-glutaryl-coenzyme A reductase inhibitors. \#: full analysis set; ๆ : patients with severe obstructive lung disease were excluded from the study; ${ }^{+}$: creatinine clearance $30-60 \mathrm{~mL} \cdot \mathrm{min}^{-1}$, creatinine clearance $<30 \mathrm{~mL} \cdot \mathrm{min}^{-1}$ was excluded

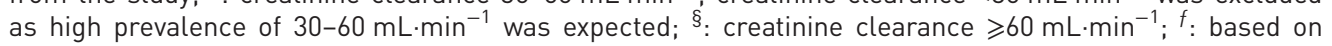
investigator reporting and systemically queried.

arterial pressure (mPAP) were observed in both treatment groups. PVR at rest at week 12, expressed as a percentage of baseline (geometric means), was similar in both treatment groups: $66.3 \%$ (95\% CI 56.2-78.3) for macitentan and $71.2 \%$ (95\% CI 51.4-98.8) for placebo. The treatment effect at week 12 (ratio of geometric means (macitentan/placebo)) was 0.93 (95\% CI 0.64-1.36) - i.e. a mean percentage reduction of $-7 \%(95 \%$ CI $-36-26 \%)$ on macitentan compared with placebo. For cardiac index, the mean treatment effect at week 12 for macitentan versus placebo was $0.4 \mathrm{~L} \cdot \mathrm{min}^{-1} \cdot \mathrm{m}^{-2}$ (95\% CI $\left.0.1-0.7\right)$. No change in PAWP or mRAP was observed in either treatment group. The changes from baseline to week 12 in other haemodynamic parameters and in echocardiographic parameters (table S7) were also similar between the treatment groups. To account for missing data at week 12, the parameters were evaluated with the use of imputation, the results were consistent with those of the main analyses (table S8).

\section{NT-proBNP}

At week 12, NT-proBNP expressed as a percentage of baseline (geometric means) was 91.6\% (95\% CI 72.4-115.8; $\mathrm{n}=25)$ in the macitentan group and $118.9 \%$ (95\% CI 92.5-152.8; $\mathrm{n}=26$ ) in the placebo group (figure 2). NT-proBNP levels were reduced at week 12 in the macitentan group versus placebo group, with 
TABLE 2 Baseline functional and haemodynamic characteristics

\begin{tabular}{|c|c|c|c|}
\hline & Macitentan & Placebo & Total \\
\hline Subjects $n$ & 31 & 32 & 63 \\
\hline \multicolumn{4}{|l|}{ NYHA functional class $\mathrm{n}(\%)$} \\
\hline II & $5(16.1)$ & 10 (31.3) & $15(23.8)$ \\
\hline III & 26 (83.9) & 22 (68.8) & $48(76.2)$ \\
\hline Median (IQR) 6MWD m & $300(216-435)$ & 305 (207-380) & $300(215-410)$ \\
\hline Median (IQR) NT-proBNP pg $\cdot \mathrm{mL}^{-1}$ & $1458(830-2700)$ & $1756(992-3503)$ & $1515(959-2921)$ \\
\hline $\begin{array}{l}\text { Median (IQR) pulse rate }{ }^{\#} \text { beats } \cdot \mathrm{min}^{-1} \\
\text { Median (IQR) blood pressure }\end{array}$ & $80.0(71.0-84.0)$ & $74.5(62.0-82.0)$ & $77.0(67.0-84.0)$ \\
\hline $\begin{array}{l}\text { Median (IQR) blood pressure" } \mathrm{mmHg} \\
\text { Systolic blood pressure }\end{array}$ & $129.0(120.0-138.0)$ & $133.0(119.0-147.5)$ & $130.0(120.0-140.0)$ \\
\hline Diastolic blood pressure & $77.0(70.0-87.0)$ & $72.0(69.0-80.0)$ & $75.0(70.0-83.0)$ \\
\hline \multicolumn{4}{|l|}{ Median (IQR) haemodynamic parameters } \\
\hline PVR dyn.s.cm ${ }^{-5}$ & $450.0(296.0-590.0)$ & $483.5(362.0-738.5)$ & $462.0(341.0-695.0)$ \\
\hline mPAP mmHg & $44.0(40.0-54.0)$ & $48.5(38.5-53.5)$ & $47.0(40.0-54.0)$ \\
\hline $\mathrm{mRAP} \mathrm{mmHg}$ & $13.0(10.0-17.0)$ & $12.5(10.0-16.5)$ & $13.0(10.0-17.0)$ \\
\hline PAWP mmHg & $20.0(18.0-21.0)$ & $20.0(16.0-23.0)$ & $20.0(17.0-22.0)$ \\
\hline TPR dyn $\cdot \mathrm{s} \cdot \mathrm{cm}^{-5}$ & $762.0(571.0-1143.0)$ & $882.5(664.5-1191.0)$ & $813.0(591.0-1158.0)$ \\
\hline Cardiac index $L \cdot \min ^{-1} \cdot \mathrm{m}^{-2}$ & $2.40(2.10-3.00)$ & $2.20(1.90-2.60)$ & $2.35(1.90-2.70)$ \\
\hline Cardiac output L. $\mathrm{min}^{-1}$ & $4.90(3.70-5.80)$ & $4.15(3.80-5.05)$ & $4.60(3.70-5.60)$ \\
\hline TPG mmHg & $27.0(21.0-33.0)$ & $27.5(21.5-33.5)$ & $27.0(21.0-33.0)$ \\
\hline $\mathrm{DPG} \mathrm{mmHg}$ & $10.0(8.0-15.0)$ & $10.0(8.0-13.5)$ & $10.0(8.0-14.0)$ \\
\hline Mixed venous oxygen saturation $\%$ & $72.0(61.0-73.0)$ & $61.0(49.0-65.0)$ & $64.5(59.0-72.0)$ \\
\hline
\end{tabular}

NYHA: New York Heart Association; 6MWD: 6-min walk distance; IQR: interquartile range; NT-proBNP: $\mathrm{N}$-terminal pro-brain natriuretic peptide; PVR: pulmonary vascular resistance; mPAP: mean pulmonary arterial pressure; mRAP: mean right atrial pressure; PAWP: pulmonary arterial wedge pressure; TPR: total peripheral resistance; TPG: transpulmonary pressure gradient; DPG: diastolic pressure gradient. ${ }^{\#}$ : data missing for four patients in the macitentan group; ": data missing for three patients in the macitentan group.

a treatment effect (ratio of geometric means) of 0.77 (95\% CI 0.55-1.08), indicating a non-significant mean percentage reduction of $23 \%(-45-7 \%)$ on macitentan versus placebo.

\section{MWD}

Mean (SD) change from baseline to week 12 in 6 MWD was $-5.9 \mathrm{~m}(71.8)$ in the macitentan group ( $\mathrm{n}=22)$ and $8.4 \mathrm{~m}(76.4)$ in the placebo group $(\mathrm{n}=25)$. The mean treatment effect for macitentan versus placebo was $-14.3 \mathrm{~m}(95 \% \mathrm{CI}-58.0-29.4)$.

Hospitalisation for worsening of HF up to EOT

Five $(16.1 \%)$ patients in the macitentan group and two $(6.3 \%)$ in the placebo group were hospitalised for worsening of HF up to the EOT (treatment difference 9.9\% (95\% CI -15.1-33.3)). All hospitalisations for worsening of HF were associated with a main study end-point event.

TABLE 3 Main safety end-point: proportion of patients with significant fluid retention or NYHA functional class worsening

Macitentan Placebo Treatment effect \% $\quad$ p-value ${ }^{\pi}$
$(95 \% \mathrm{CI})^{\#}$

\begin{tabular}{|c|c|c|c|c|}
\hline Subjects $n$ & 31 & 32 & & \\
\hline Significant fluid retention or worsening in NYHA functional class from baseline ${ }^{+}$ & $7(22.6)$ & $4(12.5)$ & $10.08(-15.07$ to 33.26$)$ & 0.34 \\
\hline Significant fluid retention & $7(22.6)$ & $3(9.4)$ & $13.21(-11.96$ to 36.21$)$ & 0.18 \\
\hline Increased body weight from baseline by $\geqslant 5 \%$ or $\geqslant 5 \mathrm{~kg}$ due to fluid overload & $3(9.7)$ & $0(0)$ & & \\
\hline Worsening in NYHA functional class from baseline ${ }^{\S}$ & $1(3.2)$ & $2(6.3)$ & & \\
\hline
\end{tabular}

Data are presented as n (\%), unless otherwise stated. NYHA: New York Heart Association. Safety analysis set; ${ }^{\#}$ : difference in proportions (\%) of patients with at least one condition; ๆ: Fisher's exact test; ${ }^{+}$: patients could meet both significant fluid retention and worsening in NYHA functional class. ${ }^{\S}$ : NYHA functional class worsened from III to IV in the macitentan patient and from II to III in both placebo patients. 
TABLE 4 Treatment effect for haemodynamic parameters at week 12

Mean absolute change from baseline $(95 \% \mathrm{CI})$

\begin{tabular}{|c|c|}
\hline PVR dyn $\cdot s \cdot \mathrm{cm}^{-5}$ & $0.93(0.64-1.36)^{\#, 9}$ \\
\hline mPAP $\mathrm{mmHg}$ & $0.3(-4.3-4.9)$ \\
\hline mRAP mmHg & $0.7(-2.2-3.6)$ \\
\hline PAWP $\mathrm{mmHg}$ & $-0.3(-4.2-3.7)$ \\
\hline TPR dyn $\cdot s \cdot \mathrm{cm}^{-5}$ & $-162.2(-318.0-6$ \\
\hline Cardiac index $L \cdot \min ^{-1} \cdot \mathrm{m}^{-2}$ & $0.4(0.1-0.7)$ \\
\hline Cardiac output L.min ${ }^{-1}$ & $0.8(0.3-1.4)$ \\
\hline TPG $\mathrm{mmHg}$ & $0.7(-3.7-5.1)$ \\
\hline DPG $\mathrm{mmHg}$ & $-0.4(-4.5-3.6)$ \\
\hline Mixed venous oxygen saturation \% & $-0.4(-4.6-3.8)$ \\
\hline
\end{tabular}

PVR: pulmonary vascular resistance; mPAP: mean pulmonary arterial pressure; mRAP: mean right atrial pressure; PAWP: pulmonary arterial wedge pressure; TPR: total peripheral resistance; TPG: transpulmonary pressure gradient; DPG: diastolic pressure gradient. ${ }^{\#}$ : geometric mean $(95 \% \mathrm{CI})$ of per cent ratio week 12/baseline; ${ }^{\Uparrow}$ : ratio of geometric means (macitentan/placebo).

\section{Discussion}

MELODY-1 was the first randomised controlled trial to evaluate the effect of an ERA in patients with $\mathrm{CpcPH}$, and one of very few trials in PH-LHD to require confirmation of diagnosis by RHC.

A number of therapies used to treat $\mathrm{PAH}$, mainly those targeting the nitric oxide and endothelin pathways, have been evaluated in studies of patients with HF and PH-LHD [7-9, 12-19]. However, results have been inconsistent; although some studies have reported improvements in exercise tolerance, functional capacity and clinical status $[18,19]$, others have failed to show statistical or clinically significant improvements [7, 9]. Moreover, early worsening of HF in studies of ERAs has led to safety concerns regarding the development of oedema during treatment $[6,7]$.

In contrast to other studies of ERAs in HF, MELODY-1 focused on patients with PH-LHD with CpcPH, most of whom had a preserved ejection fraction. The patient population displayed features typical of this condition (elderly, multiple comorbidities, concomitant medications), and most patients had some degree of reduced left ventricular diastolic function, right ventricular dilatation and right ventricular systolic dysfunction. The population had more severe LHD than in some other studies of PAH-specific drugs in HF, with higher baseline NT-proBNP levels, and worse baseline haemodynamic characteristics $[14,15,17]$. Interestingly, we observed a higher rate of atrial fibrillation in contrast with previous studies.

As oedema has been identified as a safety concern with ERAs in HF populations, the main aim of this study was to investigate the safety and tolerability of macitentan in this targeted population. In MELODY-1, numerically more patients in the macitentan group experienced significant fluid retention or worsening in FC, and therefore met the main composite study end-point compared with placebo. Fluid retention was the main driver for meeting this end-point, with the excess fluid retention events in the

FIGURE 2 Percentage of baseline $\mathrm{N}$-terminal pro-brain natriuretic peptide (NT-proBNP) at week 12 . Full analysis set. Error bars represent $95 \%$ confidence interval (CI).

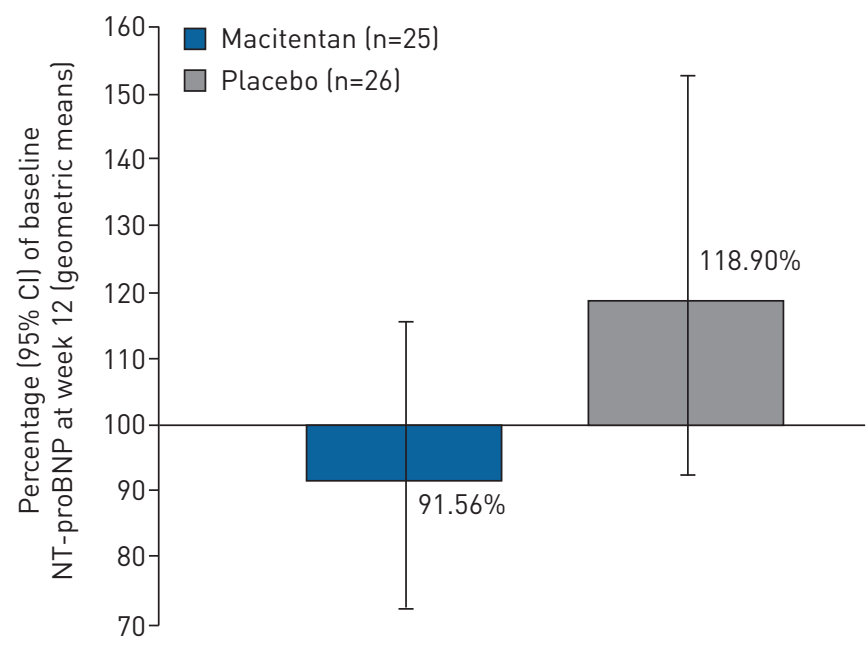


macitentan group occurring in the early part of the study. Early occurrence of fluid retention was also reported in the ENABLE study in patients with advanced systolic left HF treated with bosentan [7]. In addition, in MELODY-1, compared with placebo, a numerically higher number of macitentan-treated patients experienced serious AEs, discontinued study treatment and were hospitalised for worsening of HF. The same patients meeting the main study end-point were driving the treatment effect for these other safety parameters. More patients in the macitentan group were also reported to have haemoglobin values $<100 \mathrm{~g} \cdot \mathrm{L}^{-1}$ versus the placebo group (five versus two). Anaemia is a commonly reported side effect in clinical trials with of ERAs. The aetiology of this anaemia has not been elucidated but could be, at least in part, attributed to haemodilution.

There was no difference in PVR after 12 weeks of treatment, with a decrease of similar magnitude being observed in both treatment groups (treatment effect: 0.93 ; 95\% CI 0.64-1.36). Interestingly, there was no change in mRAP (treatment effect $0.7 \mathrm{mmHg}$; 95\% CI -4.3-4.9) or PAWP (treatment effect $-0.3 \mathrm{mmHg}$; 95\% CI -4.2-3.7), which may suggest that macitentan was not associated with worsening of left- and right-sided filling pressures at EOT. Owing to the lack of comparable invasive haemodynamic data, our results cannot be compared with previous trials studying the effects of an ERA in the context of HF [1, 2, 6-8]. It is also intriguing that we observed improvements in PVR, TPG, DPG and mPAP in the placebo group, despite no change in PAWP. Although the reason for such observations remains unclear, similar observations have been previously reported - in a study comparison of sildenafil versus placebo in patients with heart failure with preserved ejection fraction, improvements in pulmonary haemodynamics including PVR, mPAP and PAWP were reported in the placebo arm [17].

NT-proBNP is a well-established biomarker of cardiovascular response reflecting cardiac load and left ventricular wall stress [20]. In HF, elevated and increasing levels of NT-proBNP are associated with severe symptoms, fluid retention and worse prognosis [21], while a decrease is associated with improved outcomes [22]. In contrast to two recent randomised trials in HF [14, 17], we observed a non-significant decrease of $23 \%$ in NT-proBNP levels in the macitentan group (treatment effect 0.77 ; 95\% CI $0.55-1.08$ ). Although the clinical relevance of this observation is unclear, and whether there was any influence from changes in diuretics was not investigated, our findings suggest that the favourable changes in NT-proBNP occurred without worsening in cardiac filling pressures.

Limitation in exercise capacity is a key manifestation of chronic HF [23]. The 6MWD, a measure of exercise capacity, has been used in many PAH studies to assess treatment efficacy [24-26]. We observed a trend toward a decrease in the 6MWD with macitentan (treatment effect $-14.3 \mathrm{~m} ; 95 \% \mathrm{CI}-15.1-33.3$ ) that occurred in the absence of resting haemodynamic worsening. Moreover, the extent to which weight increase and oedema impacted the 6MWD remains unclear.

The study should be interpreted in the context of its limitations. It was a pilot study designed to explore safety, with a small study population and an absence of formal power calculations. The sample size makes this study sensitive to interpatient variability. In addition, there was a large proportion of missing data for some exploratory variables, mainly due to premature discontinuations. The 12 -week duration was short, and a longer follow-up period would be required to observe any cardiac structural changes by echocardiography. Nevertheless, this study is the first to explore the role of a therapeutic intervention in patients with post- and pre-capillary $\mathrm{PH}$. There are no data to support the utilisation of $\mathrm{PAH}$-specific drugs in $\mathrm{PH}$-LHD, leading to a class III recommendation for their use in the ESC/ERS guidelines [2]. The aim of our study was to generate more evidence about the potential benefit of PAH drugs (ERAs) in patients with post- and pre-capillary $\mathrm{PH}$ in the setting of an appropriately designed study in order to address this area of unmet need. The data remind us that as of yet, we do not have an effective therapy for this patient population.

\section{Conclusion}

In this phase II exploratory study, the patient population with $\mathrm{CpcPH}$ presented with advanced and severe HF. Macitentan was associated with a numerically higher incidence of significant fluid retention compared with placebo within the first 4 weeks and there appears to be no evidence of any increase in cardiac filling pressures at EOT.

\section{Acknowledgements}

Medical writing assistance was provided by Lynda McEvoy, funded by Actelion Pharmaceuticals Ltd. The authors wish to thank the investigators for their participation in the study.

\section{References}

1 Vachiery JL, Adir Y, Barbera JA, et al. Pulmonary hypertension due to left heart diseases. J Am Coll Cardiol 2013; 62: Suppl., D100-D108.

2 Galie N, Humbert M, Vachiery JL, et al. 2015 ESC/ERS Guidelines for the diagnosis and treatment of pulmonary hypertension: The Joint Task Force for the Diagnosis and Treatment of Pulmonary Hypertension of the European 
Society of Cardiology (ESC) and the European Respiratory Society (ERS): Endorsed by: Association for European Paediatric and Congenital Cardiology (AEPC), International Society for Heart and Lung Transplantation (ISHLT). Eur Heart J 2016; 37: 67-119.

3 Rosenkranz S, Gibbs JS, Wachter R, et al. Left ventricular heart failure and pulmonary hypertension. Eur Heart J 2016; 37: 942-954.

4 Hoeper MM, Lam CS, Vachiery JL, et al. Pulmonary hypertension in heart failure with preserved ejection fraction: a plea for proper phenotyping and further research. Eur Heart J 2016; 38: 2869-2873.

5 Cody RJ, Haas GJ, Binkley PF, et al. Plasma endothelin correlates with the extent of pulmonary hypertension in patients with chronic congestive heart failure. Circulation 1992; 85: 504-509.

6 Pousset F, Isnard R, Lechat P, et al. Prognostic value of plasma endothelin-1 in patients with chronic heart failure. Eur Heart J 1997; 18: 254-258.

7 Packer M, McMurray JJV, Krum H, et al. Long-term effect of endothelin receptor antagonism with bosentan on the morbidity and mortality of patients with severe chronic heart failure: primary results of the ENABLE Trials. JACC Heart Fail 2017; 5: 317-326.

8 Luscher TF, Enseleit F, Pacher R, et al. Hemodynamic and neurohumoral effects of selective endothelin A (ET(A)) receptor blockade in chronic heart failure: the Heart Failure ET(A) Receptor Blockade Trial (HEAT). Circulation 2002; 106: 2666-2672.

9 Anand I, McMurray J, Cohn JN, et al. Long-term effects of darusentan on left-ventricular remodelling and clinical outcomes in the EndothelinA Receptor Antagonist Trial in Heart Failure (EARTH): randomised, double-blind, placebo-controlled trial. Lancet 2004; 364: 347-354.

10 Actelion Pharmaceuticals. Opsumit prescribing information. 2016. http://opsumit.com/opsumit-prescribinginformation.pdf Date last accessed: May, 2017.

11 Pulido T, Adzerikho I, Channick RN, et al. Macitentan and morbidity and mortality in pulmonary arterial hypertension. N Engl J Med 2013; 369: 809-818.

12 Califf RM, Adams KF, McKenna WJ, et al. A randomized controlled trial of epoprostenol therapy for severe congestive heart failure: the Flolan International Randomized Survival Trial (FIRST). Am Heart J 1997; 134: 44-54.

13 Redfield MM, Chen HH, Borlaug BA, et al. Effect of phosphodiesterase-5 inhibition on exercise capacity and clinical status in heart failure with preserved ejection fraction: a randomized clinical trial. JAMA 2013; 309: 1268-1277.

14 Bonderman D, Ghio S, Felix SB, et al. Riociguat for patients with pulmonary hypertension caused by systolic left ventricular dysfunction: a phase IIb double-blind, randomized, placebo-controlled, dose-ranging hemodynamic study. Circulation 2013; 128: 502-511.

15 Bonderman D, Pretsch I, Steringer-Mascherbauer R, et al. Acute hemodynamic effects of riociguat in patients with pulmonary hypertension associated with diastolic heart failure (DILATE-1): a randomized, double-blind, placebo-controlled, single-dose study. Chest 2014; 146: 1274-1285.

16 ClinicalTrials.gov. Phosphodiesterase Type 5 Inhibition With Tadalafil Changes Outcomes in Heart Failure (PITCH-HF). https://clinicaltrials.gov/ct2/show/NCT01910389?term=NCT01910389\&rank=1. Date last accessed: May 2017. Date last updated: May 4, 2015.

17 Hoendermis ES, Liu LC, Hummel YM, et al. Effects of sildenafil on invasive haemodynamics and exercise capacity in heart failure patients with preserved ejection fraction and pulmonary hypertension: a randomized controlled trial. Eur Heart J 2015; 36: 2565-2573.

18 Zile MR, Bourge RC, Redfield MM, et al. Randomized, double-blind, placebo-controlled study of sitaxsentan to improve impaired exercise tolerance in patients with heart failure and a preserved ejection fraction. JACC Heart Fail 2014; 2: 123-130.

19 Guazzi M, Vicenzi M, Arena R. Phosphodiesterase 5 inhibition with sildenafil reverses exercise oscillatory breathing in chronic heart failure: a long-term cardiopulmonary exercise testing placebo-controlled study. Eur $J$ Heart Fail 2012; 14: 82-90.

20 Al-Meslmani BM, Fahoum SK, Shamia MG. NT-proBNP in monitoring treatment of patients with congestive heart failure. Clin Lab 2007; 53: 35-39.

21 Ponikowski P, Voors AA, Anker SD, et al. 2016 ESC guidelines for the diagnosis and treatment of acute and chronic heart failure. Rev Esp Cardiol (Engl) 2016; 69: 1167.

22 Masson S, Latini R, Anand IS, et al. Prognostic value of changes in N-terminal pro-brain natriuretic peptide in Val-HeFT (Valsartan Heart Failure Trial). J Am Coll Cardiol 2008; 52: 997-1003.

23 Pina IL, Apstein CS, Balady GJ, et al. Exercise and heart failure: a statement from the American Heart Association Committee on exercise, rehabilitation, and prevention. Circulation 2003; 107: 1210-1225.

24 Fritz JS, Blair C, Oudiz RJ, et al. Baseline and follow-up 6-min walk distance and brain natriuretic peptide predict 2-year mortality in pulmonary arterial hypertension. Chest 2013; 143: 315-323.

25 Provencher S, Sitbon O, Humbert M, et al. Long-term outcome with first-line bosentan therapy in idiopathic pulmonary arterial hypertension. Eur Heart J 2006; 27: 589-595.

26 Benza RL, Gomberg-Maitland M, Naeije R, et al. Prognostic factors associated with increased survival in patients with pulmonary arterial hypertension treated with subcutaneous treprostinil in randomized, placebo-controlled trials. J Heart Lung Transplant 2011; 30: 982-989. 